\title{
Parâmetros da produção in vitro de embriões da raça Sindi
}

\author{
Raquel Rodrigues Costa Mello(1), Marco Roberto Bourg de Mello(1), \\ Sabrina Luzia Gregio de Sousa ${ }^{(2)}$ e Joaquim Esquerdo Ferreira(1)
}

\begin{abstract}
(1)Universidade Federal Rural do Rio de Janeiro (UFRRJ), Instituto de Zootecnia, Departamento de Reprodução e Avaliação Animal, BR-465, Km 7, CEP 23890-000 Seropédica, RJ, Brasil. E-mail: raquelmello@ufrrj.br, mmello@ufrrj.br, esquerdovet@gmail.com (2)UFRRJ, Instituto de Zootecnia, Departamento de Produção Animal, BR-465, Km 7, CEP 23890-000 Seropédica, RJ, Brasil. E-mail: sgregio@hotmail.com
\end{abstract}

\begin{abstract}
Resumo - O objetivo deste trabalho foi avaliar os efeitos da doadora, estação do ano, do touro e do tipo de sêmen, na produção in vitro de embriões da raça Sindi (Bos indicus). Para isso, avaliaram-se 434 sessões de aspiração folicular in vivo (OPU), realizadas em 152 doadoras, com a utilização de sêmen sexado e não sexado de 22 touros. Analisaram-se as seguintes variáveis: doadora; idade da doadora; estação do ano; touro; tipo de sêmen; oócitos recuperados; viáveis e degenerados; e as taxas de clivagem e de blastocistos. Observaram-se efeitos da doadora e do touro sobre os oócitos recuperados, viáveis e degenerados e sobre a taxa de blastocisto. $\mathrm{O}$ ano foi dividido em estações chuvosa, de outubro a março, e seca, de abril a setembro. Na estação chuvosa, a proporção de oócitos viáveis aumentou, enquanto a de degenerados diminuiu. As doadoras com menos de seis anos tiveram maior proporção de oócitos viáveis e menor, de degenerados. O sêmen não sexado obteve as melhores taxas de clivagem e de blastocistos. Doadoras de até seis anos apresentam a maior proporção de oócitos viáveis, e o maior número de oócitos viáveis é produzido na estação chuvosa. O sêmen não sexado resulta em melhores taxas de clivagem e de blastocistos.
\end{abstract}

Termos para indexação: Bos indicus, doadora, OPU, sazonalidade, sêmen.

\section{Parameters of in vitro embryo production of the Sindhi breed}

\begin{abstract}
The objective of this work was to evaluate the effects of donor, season of the year, sire, and type of semen on in vitro embryo production of the Sindhi breed (Bos indicus). To this end, 434 in vivo ovum pick up (OPU) sessions, performed in 152 oocyte donors, were evaluated using sex-sorted or unsorted semen from 22 sires. The following variables were analyzed: donor; age of the donor; season of the year; sire; type of semen; recovered, viable, and degenerated oocytes; and cleavage and blastocyst rates. The effects of the donor and sire were observed on recovered, viable, and degenerated oocytes, and on blastocyst rates. The year was split into rainy season, from October to March, and dry season, from April to September. In the rainy season, the proportion of viable oocytes increased, while the proportion of the degenerated ones reduced. Donors with less than six years of age had a higher proportion of viable oocytes and a lower proportion of degenerated ones. Unsorted semen obtained the best cleavage and blastocyst rates. Donors with up to six years of age show the greatest proportion of viable oocytes, and the greatest number of viable oocytes is produced during the rainy season. Unsorted semen shows the best rates for cleavage and blastocysts.
\end{abstract}

Index terms: Bos indicus, donor, OPU, seasonality, semen.

\section{Introdução}

A crescente necessidade de incremento da produtividade pecuária tem conduzido à aplicação de várias técnicas de manejo reprodutivo, principalmente à espécie bovina, que apresenta baixo número de descendentes e longo intervalo de gerações. Neste sentido, a produção in vitro (PIV) de embriões é uma ferramenta que vem sendo largamente utilizada no Brasil, pois apresenta maior possibilidade de multiplicação do material genético obtido de fêmeas bovinas e pode contribuir de forma decisiva, para melhorar a qualidade e a quantidade do produto final, seja carne ou leite (Gonçalves et al., 2007; Rumpf, 2007).

A aplicação da PIV em escala comercial se tornou viável após o advento da aspiração folicular in vivo (ovum pick up - OPU) e pelo aperfeiçoamento das técnicas de cultivo embrionário in vitro (Pontes et al., 2011). O número total de embriões produzidos no mundo, coletados in vivo e produzidos in vitro (PIV), 
apresentou aumento de 5,8\% entre 2008 e 2012. Entre 2012 e 2013, o aumento foi de 11,6\%, chegando ao total de 1.275.874 embriões produzidos (O Embrião, 2015). Atualmente, o Brasil consagrou-se como o líder mundial na PIV de embriões bovinos, pois o número de embriões produzidos in vitro foi de 546 mil em todo o mundo, e nosso país aparece em posição de destaque, com mais de $70 \%$ do total produzido, algo em torno de 400 mil embriões (Perry, 2014).

O principal objetivo da PIV consiste na obtenção de embriões viáveis, a partir de fêmeas saudáveis de alto valor genético e, também, daquelas que não podem mais produzir descendentes pelas técnicas convencionais, como vacas doadoras que apresentam infertilidade provocada por tratamentos com gonadotrofinas. Outra vantagem está no fato de não ser necessário o uso de hormônios para a recuperação dos oócitos, o que aumenta a vida reprodutiva das doadoras. Além disso, a fertilização in vitro de oócitos recuperados permite a utilização de touros diferentes para doadoras individuais, assim como viabiliza a utilização do sêmen sexado (Andrade et al., 2012; Silva et al., 2015).

A relação entre o diâmetro folicular e a qualidade do oócito é importante para a seleção dos folículos que serão aspirados, e o sucesso e a eficiência do programa da PIV dependem da quantidade e da qualidade dos complexos cúmulus-oócitos (CCOs) recuperados (Bols et al.,1995). Assim, a eficiência da produção de embriões, como parâmetros para qualidade do oócito, é determinada principalmente durante a fase de coleta e de maturação, enquanto a qualidade dos embriões é determinada durante a fase de cultivo in vitro (Lonergan et al., 2006). Geralmente, avaliam-se os seguintes parâmetros PIV: oócitos recuperados (CCOs obtidos a cada coleta); oócitos viáveis (CCOs classificados de acordo com as características das células do cumulus e do ooplasma em graus I, II e III); oócitos degenerados (oócitos degenerados, vacuolizados ou fragmentados, desnudos e atrésicos); taxa de aproveitamento (número de oócitos viáveis em relação aos recuperados); taxa de clivagem (número de embriões clivados em relação aos oócitos viáveis); e taxa de blastocistos (número de blastocistos em relação aos oócitos viáveis) (Silva et al., 2015).

As raças de origem indiana são de grande importância para a produção de carne e leite em ambientes de clima tropical, e algumas se destacam quanto à eficiência e à produtividade em diversos países e regiões geográficas. Entre essas raças, a zebuína Sindi (Bos indicus) apresenta aptidão leiteira, alta resistência ao calor e está presente em várias regiões da Índia, Paquistão e países da Ásia, África, Oceania e Américas. No Brasil, esta raça apresenta excelente adaptabilidade às condições adversas de manejo e clima, principalmente na região do Semiárido, no Nordeste (Faria et al., 2004; Vieira et al., 2010).

A raça Sindi responde perfeitamente aos estímulos de seleção genética quanto ao desenvolvimento ponderal, fertilidade e rusticidade, o que se reflete em eficiência reprodutiva e capacidade de produção de leite, em rebanhos criados no Brasil (Mello et al., 2014). Entretanto, apesar dos avanços da PIV para algumas raças zebuínas, ainda há poucos relatos sobre a aplicação desta técnica à raça Sindi.

O objetivo deste trabalho foi avaliar o efeito da doadora, da estação do ano, do touro e do tipo de sêmen, sobre a produção in vitro de embriões da raça Sindi.

\section{Material e Métodos}

Este trabalho foi desenvolvido por meio da coleta de dados de produção in vitro de oócitos e embriões, em sessões de aspiração folicular (OPU) realizadas entre 25 de janeiro de 2008 e 15 de janeiro de 2015. Esses dados foram fornecidos pela Empresa Comercial Sexing Technologies do Brasil (Sertãozinho, SP), Tecgene e Embryo (São José do Rio Preto, SP), por meio de fichas individuais de PIV mantidas nas Fazendas Reunidas Castilho (Novo Horizonte, SP). As informações referentes às idades dos animais foram obtidas diretamente pelo endereço eletrônico da Associação Brasileira de Criadores de Zebu (ABCZ), pelo link de acesso livre à "consulta pública de animais".

Os dados referem-se a 434 sessões de OPU, realizadas em 152 fêmeas bovinas da raça Sindi doadoras de oócitos, com idades de 2 a 24 anos, devidamente registradas na ABCZ e procedentes de duas propriedades (Fazendinha e Fazenda Lagoa, localizadas na cidade de Novo Horizonte, SP), pertencentes às Fazendas Reunidas Castilho. Estas doadoras foram mantidas em condições iguais de manejo e alimentação, com pastagem de Urochloa spp. (Syn. Brachiaria spp.), mineralização e água à vontade. 
As sessões de aspiração folicular não tiveram uma rotina específica de frequência, tendo sido realizada, em média, uma sessão de aspiração a cada 20 dias. Porém, em um ou mais meses, entre os anos de 2008 e 2015, não houve sessão de aspiração folicular em nenhuma das propriedades avaliadas, além disso, em alguns meses, houve mais de uma aspiração folicular por semana.

Avaliaram-se os seguintes fatores na PIV: doadora $(\mathrm{n}=152)$, idade da doadora (até 6 anos vs acima de 6 anos), estação do ano (chuvosa vs seca), touro $(n=22)$ e tipo de sêmen (não sexado vs sexado), quanto a seus efeitos sobre os parâmetros de produção in vitro. Estes parâmetros foram: oócitos recuperados (média \pm erropadrão), oócitos viáveis em graus I, II e III (percentagem \pm erro-padrão), oócitos degenerados em grau IV (percentagem \pm erro-padrão), taxa de clivagem (percentagem \pm erro-padrão) e taxa de blastocistos (percentagem \pm erro-padrão).

As idades das doadoras foram categorizadas em duas classes para verificação de seu efeito, de modo que a classe 1 abrangeu doadoras de até 6 anos de idade (2 a 6 anos, $n=54$ doadoras), e a classe 2, doadoras acima de 6 anos ( 7 a 24 anos, $n=98$ doadoras). Adotou-se essa divisão entre doadoras mais jovens e mais velhas porque fêmeas bovinas podem alcançar a maturidade ovariana entre 5 e 8 anos de idade (Peixoto et al., 2006). Para se verificar o efeito da estação do ano, consideraram-se as sessões OPU realizadas na estação chuvosa (entre outubro e março, 232 aspirações), e na estação seca (entre abril e setembro, 202 aspirações). Para avaliar o efeito do tipo de sêmen, consideraramse as OPUs conforme o tipo do sêmen utilizado para as fertilizações in vitro - sêmen não sexado e sexado para o cromossomo X de 22 touros puros da raça Sindi -, tendo-se utilizado diferentes partidas de sêmen de cada touro. Os efeitos individuais da doadora e do touro sobre os parâmetros de produção in vitro também foram avaliados.

Para os dados relacionados aos oócitos recuperados (total de oócitos coletados), oócitos viáveis (total de viáveis, em relação aos recuperados), oócitos degenerados (total de degenerados, em relação aos recuperados), taxa de clivagem (total de embriões clivados, em relação aos oócitos viáveis) e taxa de blastocistos (total de blastocistos, em relação aos oócitos viáveis), realizou-se a análise de variância pelo procedimento Glimmix do SAS (SAS Institute Inc.,
Cary, NC, EUA). O nível de significância utilizado para rejeitar a hipótese de nulidade foi de $5 \%$ de probabilidade.

\section{Resultados e Discussão}

Observou-se grande variabilidade na PIV quanto à produção de oócitos e embriões em doadoras da raça Sindi (Tabela 1) - tal como observado pela literatura em outras raças zebuínas -, em que algumas doadoras produziram maior número de oócitos e embriões do que outras (Pontes et al., 2011; Silva et al., 2015). Esta variabilidade dos parâmetros oócitos totais, viáveis e degenerados e embriões totais e clivados pode ter ocorrido em razão da idade, da estação do ano e do tipo de sêmen que foram avaliados no presente trabalho.

Houve efeito da doadora sobre a PIV de embriões mas não sobre a taxa de clivagem (Tabela 2), o que pode estar relacionado ao fator individual quanto à competência dos oócitos para o desenvolvimento, apesar das variações pertinentes à raça, idade e escore de condição corporal (Cushman et al., 2009). De fato, observou-se uma grande variabilidade entre as doadoras relacionada ao padrão individual de recrutamento folicular. Apesar de não ter sido realizada a contagem dos folículos, é provável que as fêmeas apresentassem diferentes quantidades de folículos para serem aspirados, já que elas se encontravam em diferentes estádios do ciclo estral (Burns et al., 2005).

O número de oócitos recuperados de uma mesma fêmea não varia entre as sessões de OPU, e há um recrutamento constante de novos folículos antrais, quando eles são aspirados, porém, com variação individual de velocidade deste recrutamento. Dayan et al. (2000) verificaram grande variação da produção de oócitos e embriões in vitro em vinte fêmeas, e a

Tabela 1. Oócitos recuperados, oócitos viáveis, oócitos degenerados, embriões clivados e blastocistos, em fêmeas da raça Sindi avaliadas de 2008 a 2015.

\begin{tabular}{lccccc}
\hline Parâmetro & Total & Mínimo & Máximo & Média & CV (\%) \\
\hline Oócitos recuperados & 11.793 & 2 & 139 & $27,2 \pm 19,9$ & 55,82 \\
Oócitos viáveis & 7.972 & 0 & 86 & $18,4 \pm 14,1$ & 22,25 \\
Oócitos degenerados & 3.821 & 0 & 53 & $8,8 \pm 7,5$ & 43,72 \\
Embriões clivados & 5.336 & 0 & 42 & $12,3 \pm 9,9$ & 49,90 \\
Blastocistos & 2.820 & 0 & 32 & $6,5 \pm 5,4$ & 66,10 \\
\hline
\end{tabular}

$\mathrm{CV}$, coeficiente de variação. 
produção de blastocistos variou entre 14,0 e 54,0\%, o que indica que, na mesma doadora, podem ocorrer diferenças de produção de oócitos recuperados, oócitos viáveis e embriões. No presente trabalho, as doadoras que produziram maior número de oócitos recuperados, oócitos viáveis e embriões, por sessão de OPU, foram as mesmas doadoras em cada sessão, o que enfatiza a repetibilidade da contagem dos folículos antrais entre as fêmeas, com efeito direto sobre a PIV de embriões.

Houve efeito da idade da doadora sobre a PIV de embriões (Tabela 2), que pode estar relacionado à influência do envelhecimento sobre as funções reprodutivas, pois, doadoras mais velhas, normalmente, apresentam menor reserva folicular ovariana por ocasião das alterações no perfil hormonal (Neves \& Marques, 2008). Fêmeas mais jovens também podem ter recrutado maior número de folículos por onda de crescimento folicular e, consequentemente, produzido maior número de oócitos recuperados e viáveis por sessão de OPU (Malhi et al., 2006).

Quanto aos embriões, observou-se maior produção em doadoras de 2 a 6 anos, quando comparadas àquelas de 7 a 24 anos (Tabela 2). Malhi et al. (2008) relatam que vacas mais velhas produziram menor número de embriões e maior proporção de oócitos não fertilizados e zigotos degenerados do que vacas mais jovens. Doadoras mais velhas apresentam maior quantidade de folículos, com menor sensibilidade ao hormônio folículo-estimulante (FSH) e, portanto, são incapazes de realizar a esteroidogênese de forma normal, o que contribuiria para a menor capacidade de desenvolvimento após a fertilização e a clivagem inicial, gerando maior percentagem de oócitos degenerados (Malhi et al., 2007). No entanto, na presente avaliação, não houve efeito da idade da doadora sobre a produção de embriões. A estação chuvosa aumentou a proporção de oócitos viáveis e diminuiu a de degenerados, o que favorece a PIV de embriões (Tabela 3). Não se observou efeito da sazonalidade sobre o número médio de oócitos recuperados nem sobre as taxas de clivagem e de blastocistos. Apesar da alta temperatura observada durante a estação chuvosa, a maior precipitação pluvial exerce efeito benéfico sobre a disponibilidade de alimentos, ao aumentar a qualidade das forragens, e faz com que os animais apresentem melhor condição nutricional e, consequentemente, produzam um maior número de oócitos na PIV. Durante a estação seca, apesar da temperatura mais amena, a incidência das chuvas é bem menor, o que contribui para a piora das pastagens e pode causar estresse nutricional às doadoras, em caso de elas terem sido basicamente

Tabela 2. Oócitos recuperados, percentagem de oócitos viáveis e degenerados e taxas de clivagem e de blastocistos, de acordo com a idade das doadoras da raça Sindi, avaliadas de 2008 a 2015.

\begin{tabular}{lllcc}
\hline Parâmetro & 2 a 6 Anos & 7 a 24 Anos & Valor de P & CV (\%) \\
\hline Oócitos recuperados & $26,90 \pm 1,34$ & $20,80 \pm 1,26$ & $0,001^{*}$ & 86,60 \\
Oócitos viáveis (\%) & $68,50 \pm 0,01$ & $64,40 \pm 0,01$ & $0,01^{*}$ & 23,94 \\
Oócitos degenerados (\%) & $31,50 \pm 0,01$ & $35,60 \pm 0,01$ & $0,01^{*}$ & 47,00 \\
Taxa de clivagem (\%) & $74,40 \pm 0,03$ & $70,00 \pm 0,02$ & 0,31 & 39,00 \\
Taxa de blastocistos (\%) & $25,00 \pm 0,01$ & $28,20 \pm 0,01$ & 0,07 & 66,62 \\
\hline
\end{tabular}

*Significativo pelo teste $\mathrm{F}$, a $5 \%$ de probabilidade. $\mathrm{CV}$, coeficiente de variação.

Tabela 3. Oócitos recuperados, percentagem de oócitos viáveis e degenerados e taxas de clivagem e de blastocistos de fêmeas da raça Sindi, de acordo com a estação do ano, de 2008 a 2015.

\begin{tabular}{lcccc}
\hline Parâmetro & $\begin{array}{c}\text { Chuvosa } \\
\text { (outubro a março) }\end{array}$ & $\begin{array}{c}\text { Seca } \\
\text { (abril a setembro) }\end{array}$ & Valor de p & $\begin{array}{c}\text { CV } \\
(\%)\end{array}$ \\
\hline Oócitos recuperados & $23,40 \pm 1,21$ & $24,00 \pm 1,43$ & 0,74 & 87,55 \\
Oócitos viáveis (\%) & $67,80 \pm 0,00$ & $64,00 \pm 0,01$ & $0,02^{*}$ & 24,00 \\
Oócitos degenerados (\%) & $32,20 \pm 0,00$ & $36,00 \pm 0,01$ & $0,02^{*}$ & 47,07 \\
Taxa de clivagem (\%) & $70,40 \pm 0,02$ & $73,62 \pm 0,03$ & 0,45 & 39,01 \\
Taxa de blastocistos (\%) & $26,80 \pm 0,01$ & $26,70 \pm 0,01$ & 0,95 & 66,89 \\
\hline
\end{tabular}

*Significativo pelo teste $\mathrm{F}$, a $5 \%$ de probabilidade. CV, coeficiente de variação. 
alimentadas em pasto durante o período de avaliação (Camargo et al., 2007; Ferreira, 2011).

No presente trabalho, não se mediram diretamente as variações sazonais sobre as características das pastagens. No entanto, esse efeito sobre as pastagens tropicais é bem documentado. Como o manejo comumente empregado para as doadoras de raças zebuínas depende da obtenção dos nutrientes das pastagens, ele está sujeito a grandes variações, pois, a suplementação volumosa e concentrada é menos intensa. Isso poderia explicar a maior proporção de oócitos viáveis obtida durante a estação chuvosa, pois as forragens tropicais, nessa época do ano, apresentam aumento da digestibilidade dos seus nutrientes, o que contribui para a obtenção de melhores índices de PIV (Fernandes et al., 2001; Dourado et al., 2012; Pereira, 2012).

Gama Filho et al. (2007) observaram efeito da sazonalidade sobre a PIV em doadoras da raça Guzerá, com interferência significativa na percentagem de embriões produzidos entre o verão e o inverno (41, 20 e 90, respectivamente). Fernandes et al. (2001) também verificaram efeito da sazonalidade sobre o número de oócitos viáveis e degenerados em doadoras da raça Nelore, o que é indicativo de que importantes modificações da função ovariana, com base na produção e qualidade dos oócitos, podem ser estimadas entre as estações. No presente trabalho, não houve efeito da sazonalidade sobre a produção de embriões, provavelmente em razão da satisfatória condição corporal das doadoras e, assim, a aspiração folicular em fêmeas Sindi pode ser feita o ano todo, com a mesma eficiência da produção de embriões in vitro, independentemente da estação chuvosa ou seca.

Houve efeito do touro sobre a taxa de blastocistos, mas não sobre a taxa de clivagem, o que indica que, no

Tabela 4. Taxas de clivagem e de blastocistos, em doadoras da raça Sindi fertilizadas com sêmen não sexado ou sexado para o cromossomo X da mesma raça, avaliadas de 2008 a 2015.

\begin{tabular}{lcccc}
\hline Parâmetro & Não & Sexado & Valor & CV \\
& sexado & & de p & $(\%)$ \\
\hline Taxa de clivagem (\%) & $76,42 \pm 0,02$ & $58,89 \pm 0,04$ & $0,005^{*}$ & 37,78 \\
Taxa de blastocistos (\%) & $27,50 \pm 0,01$ & $23,13 \pm 0,01$ & $0,02 *$ & 66,10 \\
\hline *Significativo pelo teste F, a $5 \%$ de probabilidade. CV, coeficiente de \\
variação.
\end{tabular}

momento de escolher o sêmen para FIV, alguns touros proporcionaram mais blastocistos do que outros, a partir do mesmo número de oócitos. No entanto, o parâmetro taxa de clivagem torna-se secundário, uma vez que o objetivo dos programas PIV é alcançar maior número de blastocistos, e não de clivados. Quanto ao tipo de sêmen, houve influência sobre as taxas de clivagem e blastocistos (Tabela 4) que pode estar relacionada ao processo de sexagem espermática, que comumente causa danos ao espermatozoide (Carvalho et al., 2014). Há relato de uma grande variabilidade na produção in vitro de embriões com sêmen sexado, que pode ser atribuída a touros com diferentes capacitações para a produção de embriões (Palma \& Sinowatz, 2004). Há também a possibilidade de que os espermatozoides de alguns touros capacitem mais prontamente a produção de embriões in vitro do que outros, e de que alguns animais sejam mais sensíveis aos danos causados pelo processo de sexagem (Arruda et al., 2012). É importante destacar que, nesse momento, o parâmetro clivagem torna-se secundário, uma vez que o objetivo é alcançar maior número de blastocistos e não de embriões clivados.

$\mathrm{O}$ efeito do touro e do tipo de sêmen sobre a taxa de blastocistos também foi observado ao se utilizar oócitos de vacas Nelore e Gir, fertilizados com sêmen sexado ou não sexado de três diferentes touros Holandeses (Morotti et al., 2014). Do mesmo modo, Trigal et al. (2012) confirmaram o efeito individual do touro, assim como do tipo de sêmen sobre a taxa de blastocistos, que foi maior com o sêmen não sexado do que com o sexado.

\section{Conclusões}

1. Doadoras de 2 a 6 anos da raça Sindi produzem mais oócitos recuperados e viáveis na estação chuvosa, por sessão de aspiração folicular in vivo.

2. Há efeito do touro sobre a taxa de blastocistos, mas não sobre a taxa de clivagem; e doadoras fertilizadas com sêmen não sexado apresentam melhores taxas de clivagem e de blastocistos, o que favorece o programa de produção in vitro.

\section{Agradecimentos}

À Coordenação de Aperfeiçoamento de Pessoal de Nível Superior (Capes), pela concessão de bolsa ao primeiro autor; à Fazendas Reunidas Castilho, pela 
concessão dos dados; à Letícia Del-Penho Sinedino, pelo auxílio nas análises estatísticas.

\section{Referências}

ANDRADE, G.A.; FERNANDES, M.A.; KNYCHALA, R.M.; PEREIRA JUNIOR, M.V.; OLIVEIRA, A.J.; NUNES, D.P.; BONATO, G.L.; SANTOS, R.M. Fatores que afetam a taxa de prenhez de receptoras de embriões bovinos produzidos in vitro. Revista Brasileira de Reprodução Animal, v.36, p.66-69, 2012.

ARRUDA, R.P.; CELEGHINI, E.C.C.; ALONSO, M.A.; CARVALHO, H.F.; LEMES, K.M.; SILVA, D.F.; RODRIGUEZ, S.A.F.; AFFONSO, F.J. Aspects related to the technique and the utilization of sexed semen in vivo and in vitro. Animal Reproduction, v.9, p.345-353, 2012.

BOLS, P.E.J.; VANDENHEEDE, J.M.M.; VAN SOOM, A.; KRUIF, A. de. Transvaginal ovum pick-up (OPU) in the cow: a new disposable needle guidance system. Theriogenology, v.43, p.677-687, 1995. DOI: 10.1016/0093-691X(94)00073-4.

BURNS, D.S.; JIMENEZ-KRASSEL, F.; IRELAND, J.L.H.; KNIGHT, P.G.; IRELAND, J.J. Numbers of antral follicles during follicular waves in cattle: evidence for high variation among animals, very high repeatability in individuals, and an inverse association with serum follicle-stimulating hormone concentrations. Biology of Reproduction, v.73, p.54-62, 2005. DOI: 10.1095/biolreprod.104.036277.

CAMARGO, L.S.A.; VIANA, J.H.M.; RAMOS, A.A. Developmental competence and expression of the Hsp 70.1 gene in oocytes obtained from Bos indicus and Bos taurus dairy cows in a tropical environment. Theriogenology, v.68, p.626-632, 2007. DOI: 10.1016/j.theriogenology.2007.03.029.

CARVALHO, J.O.; SARTORI, R.; DODE, M.A.N. Different ways to evaluate bovine sexed sperm in vitro. Animal Reproduction, v.11, p.199-206, 2014.

CUSHMAN, R.A.; ALLAN, M.F.; KUEHN, L.A.; SNELLING, W.M.; CUPP, A.S.; FREETLY, H.C. Evaluation of antral follicle count and ovarian morphology in crossbred beef cows: investigation of influence of stage of the estrous cycle, age, and birth weight. Journal of Animal Science, v.87, p.1971-1980, 2009. DOI: $10.2527 /$ jas.2008-1728.

DAYAN, A.; WATANABE, M.R.; WATANABE, Y.F. Fatores que interferem na produção comercial de embriões FIV. Arquivos da Faculdade de Veterinária UFRGS, v.28, p.181-185, 2000.

DOURADO, A.P.; TORRES FILHO, R. de A.; CARDOSO, E. da C.; SINEDINO, L.D.P.; GERHARDT, B.T.; GOULARD, I.L.; NOGUEIRA, L.A.G. Produção estacional de embriões in vivo em vacas da raça Gir (Bos indicus) na região Sudeste (clima tropical), Brasil. Revista Brasileira de Ciência Veterinária, v.19, p.183189, 2012. DOI: 10.4322/rbcv.2014.103.

FARIA, F.J.C.; VERCESI FILHO, A.E.; MADALENA, F.E.; JOSAHKIAN, L.A. Estrutura genética da raça Sindi no Brasil. Revista Brasileira de Zootecnia, v.33, p.852-857, 2004. DOI: 10.1590/S1516-35982004000400005.
FERNANDES, C.E.; DODE, M.A.N.; GODOY, K.; RODOVALHO, N. Efeito estacional sobre características ovarianas e produção de oócitos em vacas Bos indicus no Mato Grosso do Sul. Brazilian Journal of Veterinary Research and Animal Science, v.38, p.131-135, 2001.

FERREIRA, M.B.D. Obtenção de oócitos e produção in vitro de embriões em doadoras lactantes da raça Gir (Bos taurus indicus). 2011. 176p. Tese (Doutorado) - Universidade Estadual Paulista, Jaboticabal.

GAMA FILHO, R.V. da; FONSECA, F.A.; UENO, V.G., FONTES, R. da S.; QUIRINO, C.R.; RAMOS, J.L.G. Sazonalidade na dinâmica folicular ovariana e produção embrionária em novilhas da raça Guzerá. Brazilian Journal of Veterinary Research and Animal Science, v.44, p.422-427, 2007. DOI: 10.1590/S141395962007000600005 .

GONÇALVES, P.B.D.; BARRETA, M.H.; SANDRI, L.R.; FERREIRA, R.; ANTONIAZZI, A.Q. Produção in vitro de embriões bovinos: o estado da arte. Revista Brasileira de Reprodução Animal, v.31, p.212-217, 2007.

LONERGAN, P.; FAIR, T.; CORCORAN, D.; EVANS, A.C.O. Effect of culture environment on gene expression and developmental characteristics in IVF-derived embryos. Theriogenology, v.65, p.137-152, 2006. DOI: 10.1016/j. theriogenology.2005.09.028.

MALHI, P.S.; ADAMS, G.P.; MAPLETOFT, R.J.; SINGH, J. Oocyte developmental competence in a bovine model of reproductive aging. Reproduction, v.134, p.233-239, 2007. DOI: 10.1530/REP-07-0021.

MALHI, P.S.; ADAMS, G.P.; MAPLETOFT, R.J.; SINGH, J. Superovulatory response in a bovine model of reproductive aging. Animal Reproduction Science, v.109, p.100-109, 2008. DOI: 10.1016/j.anireprosci.2007.12.002.

MALHI, P.S.; ADAMS, G.P.; PIERSON, R.A.; SINGH, J. Bovine model of reproductive aging: response to ovarian synchronization and superstimulation. Theriogenology, v.66, p.1257-1266, 2006. DOI: 10.1016/j.theriogenology.2006.02.051.

MELLO, R.R.C.; FERREIRA, J.E.; MELLO, M.R.B. Eficiência reprodutiva e produtiva em bovinos da raça Sindi (Bos taurus indicus). Agropecuária Científica no Semi-Árido, v.10, p.23-28, 2014.

MOROTTI, F.; SANCHES, B.V.; PONTES, J.H.F.; BASSO, A.C.; SIQUEIRA, E.R.; LISBOA, L.A.; SENEDA, M.M. Pregnancy rate and birth rate of calves from a large-scale IVF program using reverse-sorted semen in Bos indicus, Bos indicus-taurus and Bos taurus cattle. Theriogenology, v.81, p.696-701, 2014. DOI: 10.1016/j.theriogenology.2013.12.002.

NEVES, M.M.; MARQUES JR., A. de P. Senescência reprodutiva feminina em mamíferos. Revista Brasileira de Reprodução Animal, v.32, p.133-140, 2008.

O EMBRIÃO. [Jaboticabal]: SBTE, v. 55, 2015. Disponível em: <http://itarget.com.br/newclients/sbte.org.br/2015/extras/ embriao55.pdf $>$. Acesso em: 17 maio 2016.

PALMA, G.A.; SINOWATZ, F. Male and female effects on the in vitro production of bovine embryos. Anatomia, Histologia, 
Embryologia, v.33, p.257-262, 2004. DOI: 10.1111/j.14390264.2004.00543.x.

PEIXOTO, M.G.C.D.; BERGMANN, J.A.G.; FONSECA, C.G.; PENNA, V.M.; PEREIRA, C.S. Effects of environmental factors on multiple ovulation of zebu donors. Arquivo Brasileiro de Medicina Veterinária e Zootecnia, v.58, p.567-574, 2006. DOI: 10.1590/S0102-09352006000400019.

PEREIRA, A. Variações climáticas ao longo do ano e resultados da PIVE em doadoras de diferentes grupos genéticos. 2012. 59p. Dissertação (Mestrado) - Universidade José do Rosário Vellano, Alfenas.

PERRY, G. Statistics of embryo collection and transfer in domestic farm animals. Embryo Transfer Newsletter, v.32, p.14-26, 2014.

PONTES, J.H.F.; STERZA, F.A.M.; BASSO, A.C.; FERREIRA, C.R.; SANCHES, B.V.; RUBIN, K.C.P.; SENEDA, M.M.. Ovum pick up, in vitro embryo production, and pregnancy rates from a large-scale commercial program using Nellore cattle (Bos indicus) donors. Theriogenology, v.75, p.1640-1646, 2011. DOI: 10.1016/j. theriogenology.2010.12.026.
RUMPF, R. Avanços metodológicos na produção in vitro de embriões. Revista Brasileira de Zootecnia, v.36, p.229-233, 2007. Suplemento especial. DOI: 10.1590/S1516-35982007001000021.

SILVA, A.P.T.B. da; MELLO, R.R.C.; FERREIRA, J.E..; MELLO, M.R.B. de. Efeito do acasalamento entre a doadora e o touro (Holandês versus Gir) na produção in vitro de embriões bovinos. Boletim de Indústria Animal, v.72, p.51-58, 2015. DOI: 10.17523/bia.v72n1p51.

TRIGAL, B.; GÓMEZ, E.; CAAMAÑO, J.N.; MUÑOZ, M.; MORENO, J.; CARROCERA, S.; MARTÍN, D.; DIEZ, D. In vitro and in vivo quality of bovine embryos in vitro produced with sex-sorted sperm. Theriogenology, v.78, p.1465-1475, 2012. DOI: 10.1016/j.theriogenology.2012.06.018.

VIEIRA, D.H.; MEDEIROS, L.F.D.; BARBOSA, C.G.; RODRIGUES, V.C.; MELLO, M.R.B.; OLIVEIRA, J.P. de. Efeitos não genéticos sobre as características reprodutivas de fêmeas da raça Nelore. II - Idade à primeira parição e intervalo de parto. Revista Brasileira de Medicina Veterinária, v.32, p.79-88, 2010.

Recebido em 19 de fevereiro de 2016 e aprovado em 21 de junho de 2016 